\title{
Analysis of CT DICOM Image Segmentation for Abnormality Detection
}

\author{
Rashmi Kulkarni ${ }^{\text {a }}$, Bhavani $\mathrm{K}^{\mathrm{a}}$ \\ ${ }^{a}$ Department of Information Science and Engineering, Dayananda Sagar College of Engineering, \\ Bengaluru-560078,India.
}

Received: 11 June 2019; Accepted: 23 August 2019; Published: 08 September 2019

\begin{abstract}
The cancer is a menacing disease. More care is required while diagnosing cancer disease. Mostly CT modality is used for Cancer therapy. Image processing techniques [1] can help doctors to diagnose easily and more accurately. Image pre-processing [2], segmentation methods [3] are used in extraction of cancerous nodules from CT images. Many researches have been done on segmentation of CT images with different algorithms, but they failed to reach $100 \%$ accuracy. This research work, proposes a model for analysis of CT image segmentation with filtered and without filtered images. And brings out the importance of pre-processing of CT images.
\end{abstract}

Index Terms: Image processing, noise, filtration, image pre-processing, segmentation, nodule extraction.

(C) 2019 Published by MECS Publisher. Selection and/or peer review under responsibility of the Research Association of Mode rn Education and Computer Science

\footnotetext{
* Corresponding author.

E-mail address: kulkarni.rashmi.25@gmail.com
} 


\section{Introduction}

Cancer is a life-threatening disease. The doctors should identify cancer nodules in the early stages. CT modality is used in cancer treatment. The medical images are acquired by scanning the patients with high $\mathrm{x}$-ray beam. These scanned medical images are saved as DICOM format.

The CT images may embedded with noises during image acquisition. Spekle noise, Gaussian noise, salt and pepper noise are common in medical images. Pre-processing of medical images is done to get more accurate results. In pre-processing of images, the filters like median, CLAHE [11], Wiener, Morphological, Gabor, Gaussian, Anisotropic Diffusion, Haar wavelet Filter are used to remove the noise and to enhance the images.

PSNR is used to find the ratio of signal strength to the corrupted signal called noise. The more the PSNR value, the more the signal strength will be. An image with good PSNR is considered as good picture.

Finding the best filters for medical images is very important. In this research work, different filters are applied on five organs like Lung, Pancrease, Bladder, Stomach and Thyriod. Analysis of images is done by taking PSNR into consideration and by visualising the images.

Segmentation is the processes, in which the region of interest can be extracted [12] from the whole image. Segmentation algorithms like Watershed, Otsu, Thresholding algorithm and many more are used for image segmentation.

Finding the cancer cells growth at the earlier stage is a good option to come out of this problem. We need to build a system which detects cancer cells at the earlier stages. By using different filtering techniques on DICOM images and accurate segmentation algorithm we need to improve the accuracy of system for finding the cancer cells. In this paper, we tried to improve PSNR value by using filters on images and to increase the accuracy of finding the cancer cells by applying appropriate segmentation technique.

\section{Literature Review}

Here, survey of some techniques used for pre-processing of CT images and its segmentation techniques is discussed [2].

Suren Makaju et al. [4] proposed a model to detect cancer nodules in CT lung images. The median and Gaussian flitters are used to smooth the image and remove speckle noise from image. Watershed algorithm is used for segmentation. Feature extraction is done on the images. Used machine learning method called Support Vector Machine (SVM) as classifier to differentiate nodule as malignant or benign [4]. Proposed model detects the cancer with 92\% accuracy [4]. Hasan Koyuncu et al. [5] proposed BFO model for image enhancement before abdominal organ \& tumor segmentation. Block Matching and 3D Filtering (BM3D) algorithm, Fast Linking Spiking Cortical Model (FLSCM) [5] and Otsu algorithm are used. PSNR values are compared and found that BFO model gives good PSNR values. Jiayong Yan et al. [6] proposed a promising method to segment the liver metastases on contrast-enhanced sequential CT images. Marker-controlled watershed transform and Fuzzy connectedness algorithm [6] are used. Ashwani Kumar Yadav et al. [7] proposed a model for segmenting Brain MRI and CT Angiography images. The pre-processed images are segmented by thresholding method. The results are calculated by two parameters. That is completeness and other one is correctness [7]. M.Jayanthi et al. [8] compared the results of segmentation algorithms for liver CT images. Median filtering technique is used to remove unwanted noise[8] in images to get accurate results. Histogram Techniques, seeded region growing method, connected component algorithm, NS based thresholding algorithms are the segmentation algorithms [8] used for liver images. The dice similarity values are compared and found that the NS based thresholding algorithm works better for liver CT images than any other. P.Arjun et al. [9] proposed an improved region growing algorithm to enhance the segmentation of the 
liver from abdominal CT images[9]. Gaussian filter to remove Gaussian noise. Thresholding segmentation algorithms are applied. . K-means clustering algorithm[9] is used to classify the dataset. An improved region growing algorithm [9] partitioned the image from overlapped image to non-overlapped image. The results are compared with traditional region growing algorithm and K-means clustering. It is found that an improved region growing algorithm is having high accuracy of $97.04 \%$ where as traditional region growing is $86.03 \%$ and $\mathrm{K}$-means clustering is $87.52 \%$ [9].

Analysing this survey, the accuracy of current model are good but not satisfactory. The current researches used jpeg format of CT images. The accuracy can be achieved 100\% if the pre-processing of images is done properly. In this work, proposed a model to analyse the filters for filtration of CT images and to analyse the results of segmentation with filter and segmentation without filter. DICOM format images are used as input dataset.

\section{Proposed Model}

According to survey, it is found that pre-processing for CT image is very necessary to get the more accurate results. In proposed system, before going for segmentation of CT images, pre-processing of images[2] is done by filtering techniques. The proposed system consists of three phases. In first phase, analysis of filters on CT images is done by filtering the images with 8 different filters, and found out the best filter to remove noise in CT images. In second phase, analysis of segmentation is done on filtered CT image and on non-filtered CT image. Nodules are extracted. In third phase, the results of filtered segmentation and non-filtered segmentation are compared and analyzed.

\subsection{Type conversion}

Firstly, the CT DICOM images are converted from matrix scale to gray scale images. It converts the images with intensity between 0 to 1 . That is from black (0) to white (1).

\subsection{Image pre-processing}

In CT images, the noises are implanted while acquiring the images. The noises like speckle noise, Gaussian noise, salt and pepper noise are common. In this work , 8 different filters like Median Filter, CLAHE filter[10], Wiener Filter, Morphological Filter, Gabor filter, Gaussian Filter, Anisotropic Diffusion filter, Haar wavelet Filter are applied on Lung, Pancrease, Bladder, Stomach and Thyroid CT DICOM images. The filtered images are compared by considering their PSNR values and by visualising the images. The filter which results into more clarity image with good PSNR values is selected for CT image pre-processing. Here, the median, Gaussian and CLAHE filter gives the better results. So these filters are considered for pre-processing of CT images.

\subsection{Segmentation}

This process locates objects or boundaries which help in acquiring the region of interest in the image [12]. It helps to segment the particular part of the organ in which we are interested in. In this work, Otsu segmentation algorithm [13] is applied on the filtered and non-filtered images. Otsu algorithm classifies the images using threshold values. 


\subsection{Nodule extraction}

Finally, the cancer nodules are extracted [14] from the organ. The nodule counts of filtered segmented image and nodule counts of non-filtered segmented image are compared.

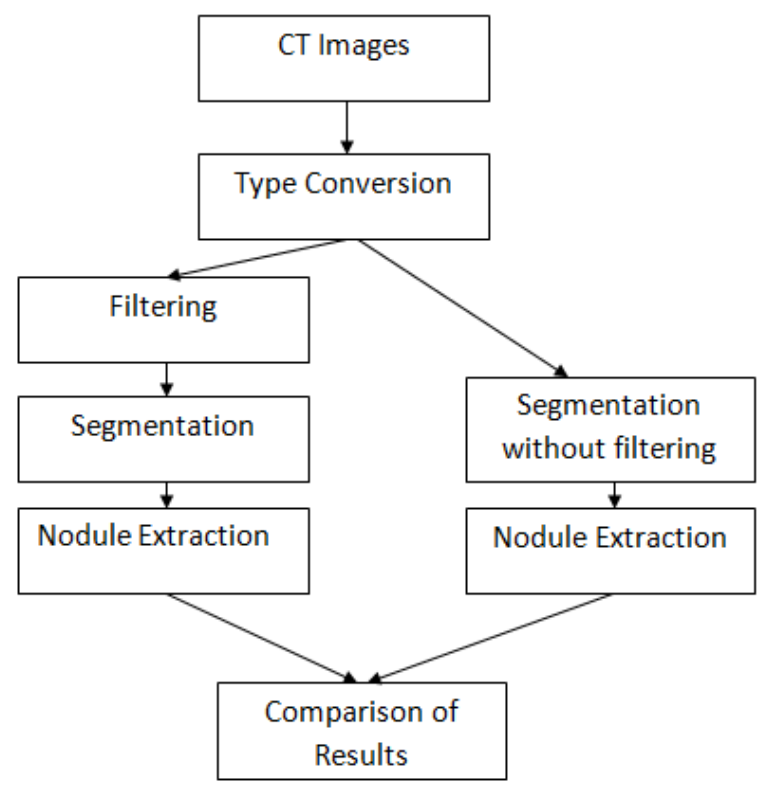

Fig. 1. Architecture of proposed system

\section{Implementation}

For implementation, the patient's real CT scan images are taken from LIDC (Lung Image Database Consortium image collection)[16] and TCIA(The Cancer Imaging Archive)[17]. LIDC is a web-accessible international resource for development, training, and evaluation of computer-assisted diagnostic (CAD) methods for lung cancer detection and diagnosis [16]. In this work the images are used are in DICOM format. The DICOM images will be of size $512 * 512$. DICOM format contains the metadata. It contains patient's details like patient's ID, age, sex, acquisition value, date of scan etc.

The proposed model is implemented in MATLAB R2014a. MATLAB is one of the tools for research development and analysis [18]. The DICOM viewer is used for analyzing the CT images. The patient details are seen through DICOM viewer.

In this model various filters are used on DICOM images and tested with combination of median, Gaussian and CLAHE filter. This combination is resulting good PSNR value. After that segmentation algorithm is applied on images. The accuracy of model in finding cancer cells is found out. 


\section{Results and Analysis}

In the first phase of this work, the different filters are applied on Lung, Pancrease, Bladder, Stomach and Thyroid. The median filter is used to remove salt and pepper noise. Gaussian filter is used to remove Gaussian noise. And CLAHE filter [11] is used for image enhancement. The results are analyzed and found out that the median, Gaussian and CLAHE filter can be used for image pre-processing.

In second phase of this work, the segmentation is done on filtered image and on non-filtered image by using Otsu segmentation algorithm [15]. Cancer nodules are extracted.

In the above, Fig2, 3, 4 shows the original image of a lung, segmented image of lung without filter and extraction of cancerous nodules respectively. Without filtration the nodule count resulted as 23 .

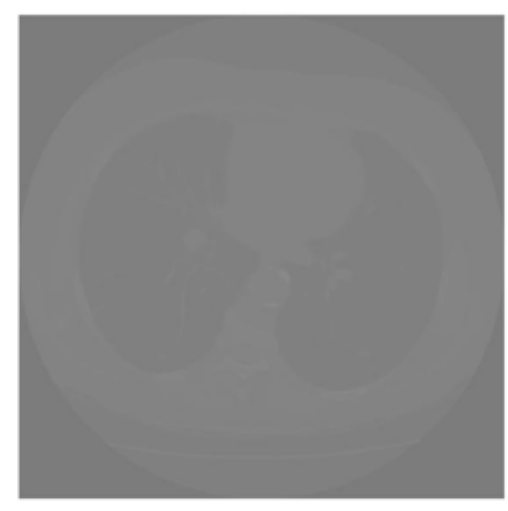

Fig. 2. Original Image

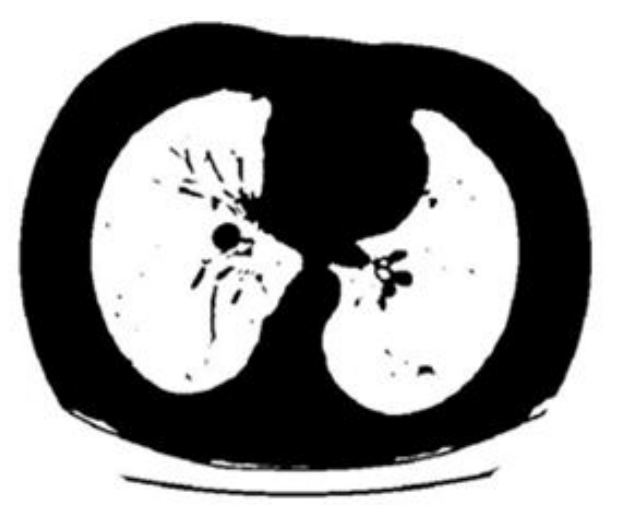

Fig. 3. Segmented without filter 


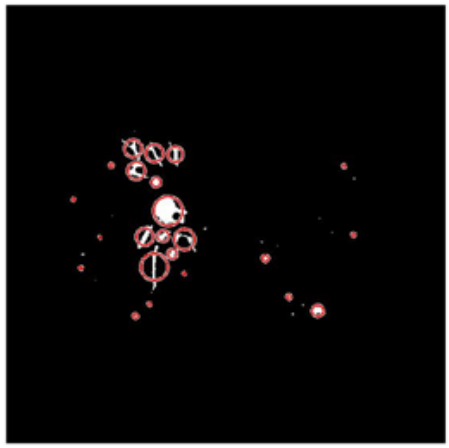

Fig. 4. Extracted Nodules with count $=23$

And Fig.5, 6, 7 represents filtered image of original lung image, segmentation with filter and nodule extraction. With filtration, the image clarity is improved and the extracted nodule count resulted as 14 . Which matched with nodule count given by LIDC data set.

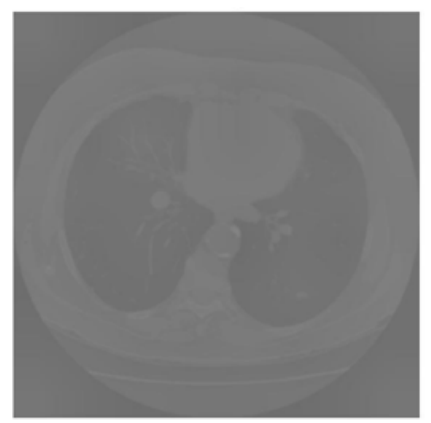

Fig. 5. Filtered Image

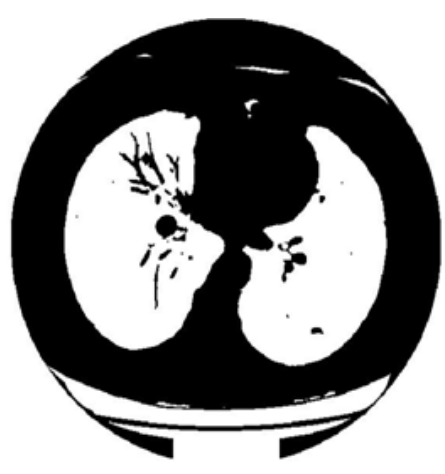

Fig. 6. Segmented with filter 


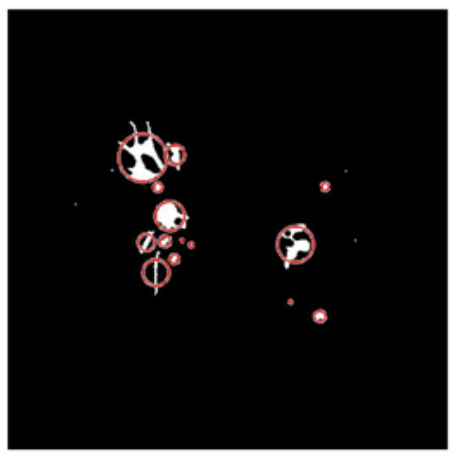

Fig. 7. Extracted Nodules with count $=14$

Here, 24 Lung images are tested and found out the accuracy. The test results are tabulated below. Table 1. Analysis of CT Images

\begin{tabular}{|l|l|l|l|l|}
\hline Images of Lung & $\begin{array}{c}\text { Expected } \\
\text { nodule } \\
\text { count }\end{array}$ & $\begin{array}{c}\text { Nodule } \\
\text { count } \\
\text { without } \\
\text { filtration }\end{array}$ & $\begin{array}{c}\text { Nodule } \\
\text { count with } \\
\text { filtration }\end{array}$ & Remark \\
\hline image1.dcm & 4 & 1 & 4 & True \\
\hline image2.dcm & 12 & 3 & 12 & True \\
\hline image3.dcm & 4 & 0 & 4 & True \\
\hline image4.dcm & 4 & 0 & 4 & True \\
\hline image5.dcm & 9 & 18 & 9 & True \\
\hline image6.dcm & 14 & 23 & 14 & True \\
\hline Image7.dcm & 2 & 0 & 2 & True \\
\hline image $8 . d c m$ & 4 & 0 & 4 & True \\
\hline image9.dcm & 11 & 28 & 11 & True \\
\hline image10.dcm & 6 & 0 & 6 & True \\
\hline image11.dcm & 17 & 24 & 17 & True \\
\hline image12.dcm & 16 & 23 & 16 & True \\
\hline image13.dcm & 34 & 60 & 32 & False \\
\hline image14.dcm & 3 & 0 & 3 & True \\
\hline image15.dcm & 2 & 16 & 2 & True \\
\hline image16.dcm & 14 & 52 & 14 & True \\
\hline image17.dcm & 1 & 7 & 1 & True \\
\hline image18.dcm & 6 & 18 & 5 & False \\
\hline image19.dcm & 5 & 23 & 5 & True \\
\hline image20.dcm & 4 & 10 & 4 & True \\
\hline image21.dcm & 4 & 48 & 4 & True \\
\hline image22.dcm & 4 & 24 & 5 & True \\
\hline image23.dcm & 2 & True \\
\hline image24.dcm & 5 & 0 & \\
\hline
\end{tabular}

True Nodules Detected images $(\mathrm{TN})=22$

False Nodules Detected images $(\mathrm{FN})=2$

Total number of images tested $=24$ 


$$
\text { Accuracy }=\frac{T N}{T N+F N}=\frac{22}{24}=0.916=91.6 \%
$$

The above results depicts that, the proposed system achieved the accuracy of $91.6 \%$ in detecting cancer nodules in patients which is higher than other models [9].

\section{Conclusion}

Though many current systems are developed for cancer detection by using segmentation, the results are not satisfactory. Therefore, proposed a system for analysis of CT image segmentation. From proposed model, it is found that, the combination of Median, Gaussian and CLAHE filters give the better results for CT images. The PSNR value is more that means more noise is reduced by using combination of 3 filters. The comparison of filtered image segmentation and non-filtered image segmentation results. Found that segmentation with filter gives the better result than the segmentation without filter, referring to the values of table.1. So pre-processing of CT DICOM image is very necessary to get accurate results of finding cancer nodules at the earlier stages. The proposed system achieved the accuracy of $91.6 \%$. Only $8.4 \%$ of test cases failed to find accurate numbers of cancer nodules in the images.

\section{Future Scope}

Although the proposed model is having accuracy of $91.6 \%$ in finding cancer cells, it is also having some limitations. The accuracy of system can be improved by using improved segmentation algorithms like watershed algorithm. And proposed system doesn't classify the stages of cancer like I, II, III, IV. So this can be improved by using supervised and unsupervised classification techniques of machine learning.

\section{References}

[1] Rafael C.Gonzalez, Richard E.Woods, "Digital Image processing”, 3rd edition ,Pearson publication,2013.

[2] Rashmi Kulkarni, Bhavani K, "A Survey on Noise Reduction and Segmentation Techniques in CT DICOM Images", International Research Journal of Engineering and Technology (IRJET), Volume: 05 Issue: 05, May 2018.

[3] M.Jayanthi, "Comparative Study of Different Techniques Used for medical image Segmentation of Liver from Abdominal CT Scan”, IEEE WiSPNET conference,2016.

[4] Suren Makaju, P.W.C. Prasad, Abeer Alsadoon, A. K. Singh, A. Elchouemi, "Lung Cancer Detection using CT Scan Images", 6th International Conference on Smart Computing and Communications, ICSCC 2017, 7-8 December 2017.

[5] Hasan Koyuncu, Rahime Ceylan, "A Hybrid Tool on Denoising and Enhancement of Abdominal CT Images before Organ \& Tumour segmentation", IEEE 37th International Conference on Electronics and Nanotechnology (ELNANO),2017.

[6] Jiayong Yan, John Q. Fang, "Segmentation of Liver Metastasis on CT Images Using the Marker-controlled Watershed and Fuzzy Connectedness Algorithms", IEEE 2016. 
[7] Ashwani Kumar Yadav, Ratnadeep Roy, Rajkumar, Vaishali, Devendra Somwanshi, "Thresholding and Morphological Based Segmentation Techniques for Medical Images”, IEEE International Conference on Recent Advances and Innovations in Engineering (ICRAIE-2016), December 23-25, 2016.

[8] M.Jayanthi, "Comparative Study of Different Techniques Used for medical image Segmentation of Liver from Abdominal CT Scan”, IEEE WiSPNET 2016.

[9] P.Arjun, M.K.Monisha, A.Mullai yarasi, G.Kavitha, "Analysis of the liver in CT images using an improved region growing technique", 2015 International Conference on Industrial Instrumentation and Control (ICIC) Col/ege of Engineering Pune, India. May 28-30,2015.

[10] Ravindra Pal Singh, Manish Dixit, "Histogram Equalization: A Strong Technique for Image Enhancement", International Journal of Signal Processing, Image Processing and Pattern Recognition Vol.8, No.8 (2015), pp.345352,2015.

[11] Brij Bhan Singh, Shailendra Patel, "Efficient Medical Image Enhancement using CLAHE Enhancement and Wavelet Fusion", International Journal of Computer Applications (0975 - 8887) Volume 167 - No.5, June 2017.

[12] Khobragade, S., Tiwari, A., Patil, C., Narke, V. , “Automatic detection of major lung diseases using Chest Radiographs and classification by feed-forward artificial neural network." IEEE International Conference on Power Electronics, Intelligent Control and Energy Systems (ICPEICES),2016.

[13] Wuli Wang, Liming Duan, Yong Wang, "Fast Image Segmentation Using Two-Dimensional Otsu Based on Estimation of Distribution Algorithm", Journal of Electrical and Computer Engineering Volume 2017, Article ID 1735176,2017.

[14] Smitha P, Shaji L, Dr. Mini M. G., “A Review of Medical Image Classification Techniques”, International Conference on VLSI, Communication \& Instrumentation (ICVCI) , Proceedings published by International Journal of Computer Applications(IJCA),2011.

[15] Hetal J. Vala, Astha Baxi, “A Review on Otsu Image Segmentation Algorithm”, International Journal of Advanced Research in Computer Engineering \& Technology (IJARCET), ISSN: 2278 - 1323, Volume 2, Issue 2, February 2013.

[16] LIDC CT DICOM image dataset retrieved from : https://wiki.cancerimagingarchive.net/display/Public/LIDC-IDRI

[17] TCIA CT DICOM image dataset of Lung, Pancrease, Bladder, Stomach, Thyroid retrieved from: https:/wiki.cancerimagingarchive.net/display/Public/Wiki

[18] Miah, M.B.A., \& Yousuf, M.A. (2015) "Detection of lung cancer from CT image using image processing and neural network." International Conference on Electrical Engineering and Information Communication Technology (ICEEICT), 2015. 


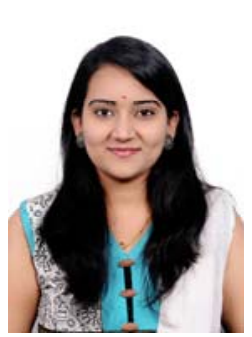

Mrs Rashmi Kulkarni received Bachelor of Engineering in Computer Science \& Engineering in2016 and Master Degree M.Tech in computer Science and Engineering in 2018 from Visvesvaraya Technological University, Karnataka. Currently she is working as Technical Mentor in private institute. Her areas of interests are Medical Image Processing, Machine Learning, and Artificial Intelligence.

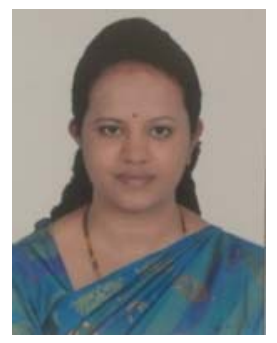

Mrs Bhavani K received Bachelor of Engineering in Computer Science \& Engineering in 2006 and Master Degree M.Tech in Computer Science and Engineering in 2009. She is currently pursuing Ph.D from Visvesvaraya Technological University, Karnataka. Her area of research include Medical image processing, Computer Vision, AI.

How to cite this paper: Rashmi Kulkarni, Bhavani K. "Analysis of CT DICOM Image Segmentation for Abnormality Detection", International Journal of Engineering and Manufacturing(IJEM), Vol.9, No.5, pp.46-55, 2019. DOI: 10.5815/ijem.2019.05.04 